\section{Huella ecológica y turismo sustentable}

DOI: $10.22403 /$ UQROOMX/TYP02/08

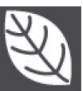

Rosa María Chávez Dagostino* Juan Luis Cifuentes Lemus Edmundo Andrade Romo Rodrigo Espinoza Sánchez

\title{
ResUMEN
}

Los investigadores en el área de turismo y diseñadores de políticas relacionadas con la actividad han llegado tarde al debate sobre el desarrollo sustentable. El análisis de la huella ecológica, ligado a la actividad turística, en el marco del enfoque estricto o "fuerte" del turismo sustentable puede permitir construir un indicador de sustentabilidad para productos turísticos en distintos niveles de agregación, la huella individual de un producto, de determinado tipo de turistas o de un centro turístico completo. Podría dar una nueva visión sobre las demandas ambientales de la actividad turística, de forma anticipada o retrospectiva y establecer qué tan sustentable es el turismo alternativo, entre otras.

Palabras Capital natural, huella ecológica, huella ecológica del turismo, CLAVE indicador de sustentabilidad, turismo sustentable. 


\section{Introducción}

El concepto y metodología para el análisis de huella ecológica (HE),' desarrollado por Wackernagel y Rees (1996) ha provocado un debate sobre varios aspectos metodológicos, de interpretación y aplicación, cuyo resultado ha sido reconocer las limitaciones propias del análisis y mejorarlo; pero en la mayoría de los casos se ha demostrado la solidez del concepto; cada vez es mejor comprendido y más utilizado.

La revista Ecological Economics (Costanza, 2000; Ayres, 2000; Deutsch et al., 2000; Rapport, 2000; Simmons et al., 2000; Templet, 2000; van Kooten y Bulte, 2000; Wackernagel y Silverstein, 2000) publicó un foro con el objetivo de estimular la discusión de tópicos relacionados con el concepto. La controversia aparece cuando los datos resultantes del cálculo de HE tratan de utilizarse como indicadores de "algo".

Se han identificado nuevas aplicaciones, utilizando HE como un sistema de medida que permite comparar la sustentabilidad entre varias opciones y determinar el mejor camino entre uso de tecnología, procesos de producción industrial, operación de empresas y diseño de escenarios, entre otras.

Si bien existe consenso en que la actividad turística es una de las "industrias" más grandes del mundo, $y$ es considerada como un importante motor de crecimiento económico, es necesario comprender las demandas a que se someten los recursos naturales con base en los que se desarrolla. Aunque la capacidad que tiene la actividad turística de degradar los ambientes naturales y culturales en los distintos destinos ha sido reconocida desde hace mucho (Hunter y Green, 1995), se ha argumentado que los investigadores en el área de turismo y los diseñadores de políticas fueron lentos en todo el mundo para responder al concepto de desarrollo sustentable y han permanecido de alguna manera ajenos al debate continuo sobre la interpretación e implicaciones de la sustentabilidad (Butler, 1999), tal vez reflejando una tendencia introspectiva de los académicos en el área de turismo.

En México, puede ejemplificarse cómo el uso del término de turismo sustentable se popularizó en planes y programas gubernamentales a finales

\footnotetext{
' Expresa en unidades de superficie la demanda sobre la productividad biológica y capacidad asimilativa de la naturaleza impuesta por el estilo de vida humano.
} 
de los 90, aun antes de ser definido en la Ley Federal de Turismo, siendo hasta el año 2004 cuando la Cámara de Senadores emitii el proyecto final de decreto de reforma a la ley en este sentido. Esta tardanza puede explicar parte de los conflictos que se han dado en todos los ámbitos en la aplicación del concepto en cuestión. En los años recientes ha emergido un número sustancial de literatura relacionada con turismo sustentable (TS), incluyendo aproximaciones de manejo y herramientas diseñadas para la integración del desarrollo de la actividad turística y la protección del ambiente natural en los destinos (Buckley, 1998; Wight, 1998), así como artículos científicos en publicaciones periódicas.

La publicación de la Guía para responsables políticos (OMT-PNUMA, 2005) cuyo objetivo es proporcionar el marco necesario para elaborar políticas orientadas a un turismo más sostenible -una "caja de herramientas" que sirva para aplicar esas políticas-, y ofrece una selección de estudios de casos, hace evidente este retraso y falta de entendimiento, pero también la necesidad urgente de virar a prácticas sustentables en la actividad turística. Al respecto señala Eugenio Yunis, jefe de Desarrollo Sostenible del Turismo en la Омт:"Las dos organizaciones tratan de alentar a todos los países a procurar que sus políticas y acciones de desarrollo y gestión del turismo se ajusten por completo a los principios de la sostenibilidad.'

Este trabajo intenta conectar conceptualmente la esferas del Ts y huella ecológica (HE), por lo que se aborda primero el debate sobre el turismo sustentable y se le relaciona en seguida con el concepto de HE aplicada al turismo (HET), finalmente, se analizan aplicaciones potenciales.

\section{El turismo sustentable y su interpretación}

Al igual que el vocablo parental de desarrollo sustentable (DS), la noción de TS permanece evasiva a una definición universalmente aceptada. Las discusiones donde se abordan interpretaciones sobre ts son más bien superficiales, sobre todo en los documentos donde se establecen políticas, pero también en muchos trabajos académicos.

Frecuentemente, el TS es explicado en términos de "encontrar un balance apropiado" entre la actividad turística, desarrollo económico, protección ambiental y la satisfacción de necesidades de turistas y habitantes locales. Con respecto a los impactos sobre el medio natural, por ejemplo, es confuso 
el grado de protección ambiental por el que se abogará (por ejemplo, la protección o la conservación de los recursos naturales) para cumplir con el precepto de sustentabilidad, por lo que pueden encontrarse empresas que presuman de sustentables cuando participan en acciones de colecta de basura en las playas.

Esta distinción es importante y es analizada con mayor frecuencia en la literatura relacionada con el DS que en la relacionada con TS. La comprensión del sentido e implicaciones de los diferentes grados de protección de los recursos naturales es un punto clave dentro del debate general sobre DS $y$ ayuda a distinguir entre los diferentes puntos de vista en el mundo, sobre la relación más apropiada entre el funcionamiento de las sociedades humanas y la utilización de los recursos naturales.

Hunter (1997) analizó los mayores puntos de debate sobre el TS, que dependen principalmente de cómo se interpretan los puntos centrales de la discusión acerca del DS: el rol del crecimiento económico en la promoción del bienestar humano, el impacto e importancia del crecimiento de la población humana, la existencia efectiva de límites ambientales para el crecimiento, la sustitución del capital natural por capital creado por el humano a través del crecimiento económico e innovación tecnológica, la interpretación diferencial sobre el estado crítico de los recursos naturales en general, la capacidad de las tecnologías para desacoplar el crecimiento económico y los efectos ambientales asociados no deseados, y el sentido de valor atribuido al mundo natural y los derechos de las especies no humanas, entre otras.

Con esta base, los investigadores en turismo se han dado a la tarea de proveer una perspectiva teórica alternativa; así, ha aparecido una clara división entre las interpretaciones “débiles" y las "fuertes" acerca de la sustentabilidad.

Las primeras se enfocan principalmente en la importancia del crecimiento económico continuo en el sector turismo y el mantenimiento suficiente de la calidad ambiental del destino, para asegurar a su vez la continuidad en la existencia de los productos turísticos y el desarrollo de nuevos productos en nuevos sitios (en este sentido puede ejemplificarse con las acciones de Fonatur en México). Esta representa una visión totalmente instrumentalista y enfocada al producto turístico, que lleva frecuentemente a prestar poca atención a la demanda de recursos naturales, donde los efectos del crecimiento sobre el ambiente natural son revisados sólo retrospectivamente, si es posible o económicamente viable. Esta interpretación de la sustentabilidad ha sido 
utilizada como medio para proveer de nuevas oportunidades al mercado turístico basado en la naturaleza.

En el otro extremo, las interpretaciones "fuertes" apelan al principio precautorio, a la planeación proactiva del desarrollo turístico y al monitoreo sistemático de cambios en el ambiente natural (capital natural). El énfasis se hace en la protección de los recursos naturales que soportan la actividad turística, donde el capital natural debe ser constante (Driml y Common, 1996) y considerarse como capital crítico (Claude, 2004). Sin embargo, surge la pregunta fundamental, como lo han señalado varios autores (Hunter, 1995; Buttler, 1999), sobre el propósito básico del pensamiento relacionado con Ts: ¿Debe el Ts crear las condiciones a fin de que la actividad turística pueda sobrevivir a largo plazo por su propio bien, o debe ser la forma en la que la actividad turística contribuya a los objetivos generales del Ds?

El DS no puede sectorizarse (por ejemplo en turismo) porque entonces pierde su sentido esencial. Esto puede considerarse como la esencia fundamental de las diferencias entre los enfoques.

Las visiones sobre el DS (tabla I) muestran el pluralismo emergente en el pensamiento académico, pero además la huella ecológica del turismo (HET) está relacionada en varios aspectos con estas ideas.

\section{El concepto de huella ecológica aplicado al turismo}

Si se elige la forma de interpretación rígida, las técnicas desarrolladas para medir la HE y su análisis, podría dar una nueva visión sobre las demandas ambientales de la actividad turística, anticipada o retrospectivamente y compararla con otras actividades. Incluso podrían establecerse los impactos causados por distintos productos turísticos y los impactos diferenciales de los distintos segmentos de turismo o más aún, de los tipos de turistas que son atraídos a los destinos turísticos.

Con todas estas nuevas aplicaciones de HE la contribución principal sería la capacidad de formular actividades turísticas, actuales o potenciales en términos de la demanda ecológica, más allá de la capacidad de carga, a veces utilizada en estudios relacionados con el turismo. Casi todas las formas en que se miden los impactos en turismo están limitados a aspectos de calidad ambiental de relevancia directa, tangible o inmediata para la existencia del 
Huella ecológica

y turismo sutentable

TABLA I.DESCRIPCIONES SIMPLIFICADAS DEL DS CON BASE EN LA VISIÓN “DÉBIL" Y “FUERTE” (Modificado de Hunter, 1997)

\section{Visiones sobre Ts}

Débil

- Se enfoca y favorece fuertemente al desarrollo de nuevos productos

- Los beneficios del turismo se asumen

- "Preciosa" vista del turismo como sector donde dominan los intereses del sector

- Mantener la actividad turística en los destinos existentes y expandirlo a nuevos

- Los productos turísticos deben mantenerse y evolucionar de acuerdo a las necesidades del mercado

- La acción ambiental se da sólo cuando es requerida o benéfica

- Más probable que se tenga un involucramiento directo en la industria
Fuerte

- Se basa en el conocimiento-precaución

- Los beneficios del turismo deben ser demostrados

- El turismo no necesariamente es una componente del DS en un área

- Ampliar la base económica si hay alta dependencia en el turismo y se compromete en la evaluación proactiva de un nuevo desarrollo turístico

- Los recursos naturales deben mantenerse, y reducirse los impactos donde sea posible, con productos diseñados con esa intención.

- Los impactos ambientales siempre se consideran como cuestión de rutina

- Más probable que se tenga un entrenamiento en disciplinas académicas ambientales

producto turístico local (Hunter, 1995). Otro tipo de demandas sobre los recursos naturales no se miden como las implicaciones energéticas, de agua, suelo, etc. Aun en el campo del Ecoturismo donde, al menos en teoría, es importante la integridad funcional de los ecosistemas, los cuestionamientos académicos ignoran el estrés impuesto por este tipo de experiencias sobre la biosfera.

La HET puede ser conceptualizada de varias formas: como un sector completo, como un componente sectorial, como un producto, como un destino, de acuerdo con el tiempo, etc. En teoría, los cálculos de la HE deberían ser para la industria turística como un todo, es decir, la suma de la demanda 
de la actividad turística sobre los recursos naturales en el planeta. Este trabajo a nivel mundial no se ha desarrollado para ningún sector. A otras escalas hay muy pocos esfuerzos relacionados con la actividad turística.

Un cálculo de este tipo puede ser una pérdida de tiempo, debido a que es una industria muy diversa. Está caracterizado por una amalgama de intercambios fragmentados, organizaciones y actividades, que involucra desde las fuentes emisoras, el evento de tránsito de turistas y los eventos en los destinos.

No obstante la complejidad de la actividad, puede mirarse a una parte del sector. Wackernagel y Yount (2000) estimaron que la HET internacional debía ser mayor a $10 \%$ de la huella planetaria dado que este mercado genera $10 \%$ de las ganancias a nivel mundial. Aunque se han hecho otros intentos, como estimar la contribución de las líneas aéreas a la emisión global de $\mathrm{CO}_{2}$ (Copeland en 1992 lo calculó en 2\%), existen formas más sencillas de abordar el tema, tal como monitorear la $\mathrm{HE}$ en el turismo a partir de productos individuales (considerados como la unidad turística básica estructural y funcional). Como ejemplo puede tomarse un "paquete todo incluido" por una semana en un centro turístico de playa. Los impactos ambientales por la demanda de recursos que causa un turista en este caso y que contribuye a elevar la HE, comienza desde el lugar emisor y se extiende a las áreas de tránsito y destino. Cada una de estas demandas pueden ser convertidas en unidades de superficie (por ejemplo, energía utilizada en el viaje convertida a cantidad de $\mathrm{CO}_{2}$ y superficie vegetal necesaria como resumidero). Es pertinente mencionar la importancia que ha adquirido la idea del impacto ambiental del transporte en general, y sobre todo considerado en el contexto de la actividad turística, con la campaña que realizan las agrupaciones Fundación Ecología y Desarrollo y Fundación Natura (2005), de concienciación sobre las emisiones de $\mathrm{CO}_{2}$ a la atmósfera durante los viajes $y$, con un mensaje en la confirmación de billetes de avión que dice: "Si te preocupa el cambio climático y quieres ayudarnos a combatirlo, calcula las emisiones de $\mathrm{CO}_{2}$ que se emitirán a la atmósfera con este viaje. Tendrás la posibilidad de ayudar a compensarlo a través de proyectos de desarrollo limpio en América Latina", permite calcular por paquetes con base en el uso de los diversos energéticos, las toneladas del gas que se producirían y el costo en euros, de las acciones para compensar dichas emisiones. 
Puede calcularse así la HE de los "paquetes" a partir de los turistas que lo compraron (por producto o per capita).

Tal vez lo más importante de todo esto sea que los datos obtenidos del análisis de HET, podrían permitir una comparación directa entre los diferentes tipos de turismo en términos del desempeño ecológico global (turismo alternativo vs. turismo masivo).

En una revisión sobre turismo sustentable, Butler (1999) argumentó que el debate sobre el turismo sustentable ha sido confundido por la tendencia de algunos investigadores, diseñadores de políticas y operadores turísticos, de igualar automáticamente el llamado turismo alternativo con lo sustentable, en tanto se refieren al turismo de masas como inherentemente no sustentable. Las alternativas de turismo son frecuentemente descritas como de pequeña escala, benignas en términos de impactos y basadas en una experiencia genuina; por el contrario, el turismo de masas, en otro extremo, involucra empresas de gran escala que atienden una gran cantidad de turistas en destinos atestados y dedicados a ofrecer "comodidades como en casa" y "caricaturas o remedos" del estilo de vida local.

Aunque existe un consenso general con lo anterior y se admite que esta última forma de actividad masiva siempre se asocia con un mayor grado de deterioro ambiental local, la HE puede proveer una visión más holística de la sustentabilidad y la demanda ambiental.

Otro tema interesante es el cálculo de la HE de los turistas. Una primera aproximación nos llevaría a pensar que los turistas conservan la huella ecológica promedio del país en que viven, sin embargo, se sabe que la demanda de recursos aumenta cuando uno se convierte en turista, comparado con el estilo de vida habitual, pero debe demostrarse y determinarse en qué sentido se amplifica esta demanda. En general, podemos esperar encontrar diferencias muy marcadas cuando los turistas de países desarrollados vacacionan en hoteles lujosos en países menos desarrollados.

Por otra parte, como lo señalan Wackernagel y Yount (2000), la HE no cuantifica propiamente al turismo y aún más, es contabilizado como parte de la demanda ecológica de los destinos, más que del país emisor. Con suficiente información sobre los productos turísticos esta demanda podría "repatriarse", haciendo aún más evidente la diferencia entre la HE de los países más desarrollados con la de los menos desarrollados. Por ejemplo, la HE del conjunto 
de viajes al año que realiza un ciudadano (tres para un norteamericano), puede equivaler a la HE completa de un ciudadano de un país pobre. Estas comparaciones pueden servir para ilustrar desde otro enfoque la brecha entre pobres y ricos del mundo, que ha de disminuirse si se plantea cumplir con el principio de equidad intrageneracional del desarrollo sustentable (WCED, 1987).

La demanda ecológica debida a la actividad turística no se mide, y cuando se toma en cuenta en los aspectos ambientales, se hace de forma retrospectiva y con la finalidad de salvaguardar el sitio en pro de la actividad turística y no de contribuir al logro de los objetivos del desarrollo sustentable.

El concepto y técnicas de HE, que han sido mejoradas y aplicadas en distintos contextos y niveles, permite hacer aproximaciones diferentes en términos de demanda ecológica global, local o regional. Provee también un medio para retomar el análisis teórico y práctico del turismo sustentable (retórica que ya se ha desgastado) y da la oportunidad de extender nuestro entendimiento del turismo en el campo del impacto ambiental.

\section{FUENTES CONSULTADAS}

Ayres, R. U. (2000). "Commentary on the utility of the ecological footprint concept". Ecological Economics, 32, 347-349.

Buckley, R. (1998). “Tools and indicator for managing tourism in parks”. Annals of Tourism Research, 25 (4), 208-2II.

Butler, R.W. (1999). "Sustainable tourism: a state-of-the-art review". Tourism Geographies, I (I), 7-25.

Claude, M. (2004). “La riqueza natural como capital crítico”. Ambiente y Desarrollo, 20 (2), I27-I 28.

Costanza, R. (2000). “The Dynamics of the ecological footprint concept”. Ecological Economics, 32, 34I-345.

Copeland, E. (1992). "The role of airlines in the tourism and environment debate". Tourism Management, 13, I I 2-I I4.

Deutsch, L. et al. (2000). "The ecological footprint: communicating human dependence on nature's work”. Ecological Economics, 32, 35I-355.

Driml, S. y M. Common (1996). "Ecological economics criteria for sustainable tourism: applications to the Great Barrier Reef and Wet Tropics 
World Heritage Areas, Australia”. Journal of Sustainable Tourism, 4 (I), 3- 16

Fundación Ecología y Desarrollo y Fundación Natura (2005). "Cero $\mathrm{CO}_{2}$, iniciativa para el cuidado del clima” [en línea], España, disponible en: <http://www.Lastminute.com y http://www.ceroco2.org> [2006, I2 de enero].

Hunter, C. (1997). "Sustainable tourism as an adaptive paradigm". Annals of Tourism Research, 24 (4), 850-867.

Hunter, C. y H. Green (1995). Tourism and the Environment: a Sustainable Relationship?. Londres/Nueva York: Routledge.

OMT-PNUMA (2005). Por un turismo más sostenible. Guía para responsables políticos. Organización Mundial del Turismo y Programa de las Naciones Unidas para el Medio Ambiente.

Rapport, W. E. (2000). "Ecological footprints and Ecosystem health: complementary approaches to a sustainable future". Ecological Economics, 32, 367-370

Simmons, C., K. Lewis y J. Barret (2000). "Two feet-two approaches: a component-based model of ecological footprinting”. Ecological Economics, 32, 375-380.

Templet, P.H. (2000). "Externalities, subsidies and the ecological footprint: an empirical analysis". Ecological Economics, 32, 38I-383

Van Kooten, G.C.y E. Bulte (2000). "The Ecological Footprint: useful science or politics?”. Ecological Economics, 32, 385-389.

Wackernagel, M. y W.E. Rees (1996). "Our Ecological Footprints: Reducing Human impact on the Earth”. Gabriola Island, B.C., Canadá: New Society Publishers.

Wackernagel, M. y J.D. Yount (2000). "Footprints for sustainability: the next steps". Environment, Development and Sustainability, 2, 21-42.

Wackernagel, M. y J. Silverstein (2000). "Big things first: focusing on the scale imperative with the ecological footprint”. Ecological Economics, 32, 39|-394.

WCED (1987). Our Common Future. Oxford: World Commission on Environment and Development/Oxford University Press.

Wight, P. (1998). "Tools for sustainability analysis in planning and managing tourism and recreation”, citado en C. M. Hall y A. Lew (eds), Sustainable Tourism: a Geographical Perspective. Harlow: Longman. 